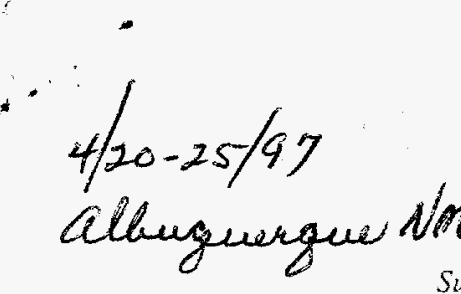

Submitted to the 1997 IEEE International Conference on Robotics and Automation

\title{
An Interactive System for Creating Object Models from Range Data based on Simulated Annealing:
}

\author{
William A. Hoff, Frederick W. Hood, Robert H. King \\ Center for Robotics and Intelligent Systems \\ Colorado School of Mines Golden, $\mathrm{CO} 80401$
}

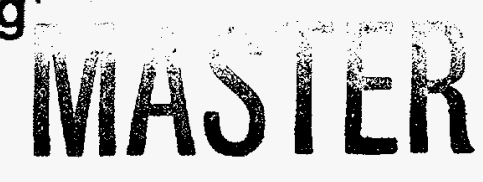

\begin{abstract}
Sensing to recognize and locate objects is a critical need for robotic operations in unstructured environments. An accurate 3-D model of objects in the scene is necessary for efficient high level control of robots. Drawing upon concepts from supervisory control, we have developed an interactive system for creating object models from range data, based on simulated annealing. Site modeling is a task that is typically performed using purely manual or autonomous techniques, each of which has inherent strengths and weaknesses. However, an interactive modeling system combines the advantages of both manual and autonomous methods, to create a system that has high operator productivity as well as high flexibility and robustness. Our system is unique in that it can work with very sparse range data, tolerate occlusions, and tolerate cluttered scenes. We have performed an informal evaluation with four operators on 16 different scenes, and have shown that the interactive system is superior to either manual or automatic methods in terms of task time and accuracy.
\end{abstract}

\section{Introduction}

In hazardous applications such as remediation of buried waste and dismantlement of radioactive facilities, robots are an attractive solution. Such environments are typically unstructured, in the sense that the types and locations of objects are not known in advance.

Control of robots and machinery for use in such operations ranges from full manual control (direct teleoperation by a human operator) to full automatic control (no input from a human operator). Between these two extremes lies a paradigm called supervisory control [1]. which allows the svatrm to perform low level tasks automatically under the supervision of a human operator. Supervisory control is a promising technique for near term operations: it retains the flexibility of human intelligence to respond to unforeseen events and combines it with the speed and accuracy of the computer for performing lowlevel tasks [2].
In order for the system to perform low level tasks under supervisory control, three-dimensional (3-D) graphical models are needed which accurately represent the location, type, shape, etc.. of objects in the scene [3]. For certain environments, blueprints and architectural drawings may exist, but these cannot be relied upon to be accurate. In unstructured environments, the 3-D site model must be created from sensor data.

The usual practice in creating object models is to physically measure their locations and then manually create them in software or with an interactive user interface. However, this is a time consuming task, and one that should be avoided in a hazardous environment. Some systems allow the locations of objects to be measured remotely, using a range inder or other sensor. However, the human operator still must manually create the object model, using the location information as a guide. Also, it is not sufficient to specify just the location of the object - the model requires the object orientation, as well as any other parameters describing its size, shape, etc.

Two dimensional (2-D) video images and/or three dimensional (3-D) range images can provide the necessary data to create object models. Past work on object modeling has been either primarily manual or primarily automatic. Manual techniques have the disadvantage of requiring a substantial amount of effort from the human operator. Automatic techniques have been limited in performance and reliability. Most automatic systems are limited to recognizing a small number of simple object models, in clean, uncluttered scenes. The generality and flexibility of current systems is very limited, especially for those that use image data (as opposed to range data). Therefore, there is a need for techniques which are more automated, but are robust and flexible in dealing with new environments.

In this paper, we describe supervisory techniques that we have developed for creating object models, that have been shown to be highly robust and flexible. These techniques are interactive; that is, they use the human to guide the sensing system in creating and updating models of objects. The motivation for using interactive

This project has been funded by the NEL University Research Consortium. The NEL is managed by Lockheed Martin Idaho Technologies Company for the U.S. Department of Energy, Idaho Operations Office, under Contract No. DE-AC07-94IDI3223 
techniques is that purely manual and purely automatic techniques both have disadvantages. However, a hybrid system has the potential to combine the best attributes of each to create a system that has high operator productivity as well as high flexibility and robustness. The strategy is similar to the use of the human in supervisory control, in which human intelligence and machine intelligence are integrated to create a more powerful system that uses the best elements from each component. The human provides high level reasoning and overall guidance; the computer provides quantitative analysis and repeatable operation. Thus, we have extended the supervisory control paradigm to sensing.

We have developed a system which uses sparse, noisy range data (about $50-100$ points per object) obtained from a stereo vision sensor. The operator can interact with the system through a combination of traded and shared control, to create models of objects in the scene. We have evaluated our system with a combination of synthetic and real scenes. In informal tests with four operators, we have shown that the supervisory system is superior (in terms of task time and accuracy) over purely manual and purely automatic modeling. Our effor is unique by virtue of the use of the interactive techniques, sparse range data collected from stereo vision and other sources, and an optimization technique called simulated annealing for fitting primitive $3-\mathrm{D}$ object models to the range data.

The rest of our paper is organized as follows: Section II covers background information related to object modeling; Section III gives an overview of our system; Section IV describes implementation details and experimental results; and Section V provides conclusions

\section{Background}

Past work on creating object models has generally fallen into the categories of primarily manual or primarily automatic. With primarily manual systems, the operator creates, sizes, and places a graphical object model to correspond to a physical object that he or she observes in the scene. In Sandia's Graphical Programming System [3], the operator can control the robot to touch an object with a probe in order to determine its position. Other systems use the operator to examine imagery obtained from sensors and create object models to fit the observed objects $[4,5]$. Other related work includes photogrammetric reconstruction from still photographs, developed by TRW and Vexcel Corp. In all these systems, the operator rather than the computer, performs the bulk of the work in creating and specifying the attributes of the model.
In the category of primarily automatic systems, much work has been done in the computer vision ficld on automatically recognizing and creating models from range data. Here, we wish to draw a distinction between techniques which just construct a surface map [6] or occupancy map [7] of the scene, and those (like ours) which fit geometric models to discrete objects in the scene. In the latter class of techniques, researchers have developed systems to fit superquadric volumetric primitives [8], generalized cylinders [9], and parametric geons [10], to range data. Hebert, et al [11] use the operator to select an initial region of interest in the image, then automatically fits a cylinder to the surface data.

Other work matches a specific object model to range data. For example, Grimson, et al matches a model derived from MRI data to laser range data [12]. Besl and McKay [13] register two 3-D shapes using the iterative closest point algorithm. Most techniques use an iterative algorithm to find a solution, either based on least squares or Kalman filtering [14].

Most previous work does not address the segmentation of the data - i.e., how to automatically distinguish points on the object from points in the background. Also, the objects must be relatively unoccluded for the model fitting to converge correctly. In typical unstructured environments, such as a drum half buried in a landfill, these techniques could not correctly create the object model. Finally, most algorithms require fairly dense range data with many points on the object of interest.

Recently, researchers have argued that fully automated systems for object recognition and modeling are currently incapable of matching the human's ability to employ background knowledge, common sense, and reasoning. One way to increase the flexibility of a computer vision system is to allow an outside entity, such as a human, to provide context and constraints to the vision system. This approach has been used successfully in the domain of overhead image interpretation. Recently, under DARPA's RADIUS project, much work has gone toward automating portions of the interpretation process, using image understanding techniques [15]. Much success has been achieved by developing interactive techniques to extract building and road models [16]. For example, an image analyst can provide an initial approximation for a building outline and let the vision system perform local optimization of the variables to fit the data. [17] Although these techniques are promising, they have been applied only to the overhead image interpretation domain (primarily 2-D). They must be modified to apply them to the robotic domain (primarily 3-D), and in which viewpoints are much more unconstrained. 


\section{DISCLAIMER}

Portions of this document may be illegible in electronic image produets. Images are produced from the best available original document. 


\section{DISCLAIMER}

This report was prepared as an account of work sponsored by an agency of the United States Government. Neither the United States Government nor any agency thereof, nor any of their employees, makes any warranty, express or implied, or assumes any legal liability or responsibility for the accuracy, completeness, or usefulness of any information, apparatus, product, or process disclosed, or represents that its use would not infringe privately owned rights. Reference herein to any specific commercial product, process, or service by trade name, trademark, manufacturer, or otherwise does not necessarily constitute or imply its endorsement, recommendation, or favoring by the United States Government or any agency thereof. The views and opinions of authors expressed herein do not necessarily state or reflect those of the United States Government or any agency thereof. 


\section{Detailed Description}

Our wsem consists of three main funcuonal elemencs: (1) a source of range data points, (2) a moxel fitting algorithm. and (3) interactive isupervisory) technique, These are discussed in the sections below

\section{A. Stereo Vision Sensor}

We have develored a stereo vision sensor for obtaining range datat that is, a computer vision system which accepts mages from multiple cameras, automatkilly matches points between the images and compute the range va triangulation. Measuring range via sterew sum is widely considered wo be a difficule problem due to the dithuly in correcty matching points between the tur mages. The resulting range values may be sparse and ambiguous (because of non-unique matches). We chose serew because (a) it is a more challenging sensor ilf our techniques work well with stereo. it is highly prothate that they will work with active sensorst. and the vereo has potental advantages of cost. stes. and

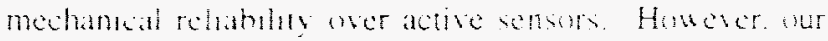
system an work whth any source of range datal and we have in bot und it with data from a structured light sensor

Our whem uses three camerats in a tronocular arrangemont The purpose of the third camera is 10 eliminate matchng errors between the first two cameras |18|. An example of a trinocular set of mages as shown in Figure 1

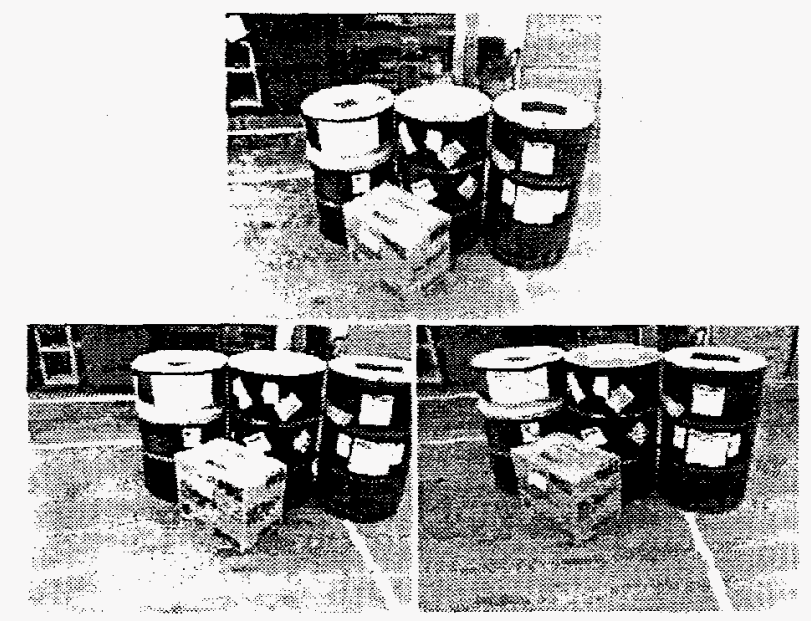

Figure 1 Top, left, and right images from stereo vision system.

The stereo vision system detects "interest" points in the left and right images, and matches them using a crosscorrelation technique. Each candidate match determines a point in $3-\mathrm{D}$. These points are checked by verifying their presence in the predicted location in the top image. Points which are not verified are climmated. Figure? shows the final range points as cross-hats werlaid on the left image.

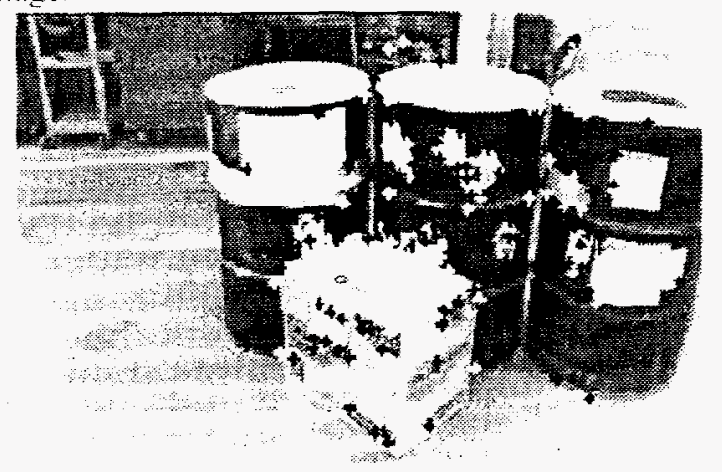

Figure 2 Final range data points (crosshairs).

We have tested the syitem on 13 real scencs and numerous synthetic scenes. On the average. the system produced 88 3-D points per seene the manually examined the and found an average of 4 hath mathes remathing

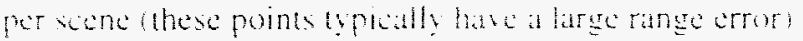

\section{B. Model Fitting Algorithm}

An algurihm has been develuped 10 it geometric primitive models to range data. The algormh iteratid: adjusts a state vector representing the parameters of the model in order to minmue the crror distances between the range data points and the nearest punt on the surface of the model. Currenty, the state tector represents only the weation and orientation $16 \mathrm{DOP}$, the model. although we are extending it to include parmeters describing sts and shape. The algorthm uses the "downhll smplex" method to search for the minmum [19]. However. there is a strong possibility that the algorthm will get stuck in a hal minima, which may be fir trom the true solution.

To avoid this, a simulated annealing algorithm [20] is built on top of the downhill simplex algorithm. The simulated annealing algorithm allows the solution an occasionally move to a point with higher (worse) emor. This usually allows the algorithm to escape local minima and eventually find the global minimum. A "temperature" parameter controls the probability of moving to a higher error state: the higher the temperature, the greater the likelihood of moving to a higher error state. The algorithm is analogous to the slow cooling of a metal. which allows the atoms to arange themselves into a crystal that is the minimum energy state (hence the name "simulated annealing"). We roduced the temperature at a constant rate; other reduction schedules are possible [2]]. 
An important issue is the definition of the error function. One possibility is to define the error as the sum-of-squared distances between the range data points and the nearest point on the surface of the model. With this approach, points with large distances dominate the total error score. This has the problem that outliers (points which do not belong to the model) greatly affect the resulting fit. A error function is needed in which outliers do not affect the fit. This error function is defined as

$$
\text { errorscore }=\sum_{i=1}^{\# p r s}-G / r_{i}
$$

where $r_{i}=$ distance from the $i^{\text {th }}$ point to the model and $G$ is a constant. As $r_{i}$ approaches infinity, the contribution of the point to the error score goes to zero. As $r$ approaches zero, the contribution of the point to the error score would approach negative infinity; however, we limit the minimum score to $-G / D_{\mathrm{MN}}$, where $\mathrm{D}_{\mathrm{MIN}}$ is the estimated uncertainty in position of our data points ( $1 \mathrm{~cm}$ ).

This error function has the same form as the potential energy resulting from a gravitational or electrostatic force. In fact, each point may be considered to exert a force proportional to $1 / \mathrm{r}^{2}$ on the model, where $r$ is the distance to the model surface. Very close points exert a large force; and very distant points exert a negligible force. Thus, outliers have a negligible effect on the overall fit.

In experimentation to date, we have found that the model fitting algorithm converges to a minimum in several thousand iterations, which takes about 10-15 seconds on a Silicon Graphics Indigo 2 workstation (this also includes the time required to draw 3-D graphics). In some cases, this is the true solution (global minimum), but in other cases it is an incorrect solution (local minimum). In the latter cases, the user must intervene (sce next section) in order to reach the correct solution.

\section{User Interaction Techniques}

We have developed software to allow the user to visualize and interactively fit 3-D graphical models on the Silicon Graphics workstation. The software displays range data points and geometric primitives (spheres, cones, cylinders, and parallelepipeds) as overlays on top of background images from the stereo cameras. These objects can be displayed in normal 2-D mode or in 3-D mode, using stereo viewing glasses. For an input device, the operator can use the usual 2-D mouse, or a 6 degreeof-freedom (DOF) mouse.

The user first selects a geometric primitive (model) to fit to the data points. The user can manipulate the size and shape of the model with a wire-frame "handle-box" surrounding the model, or by entering data into a text box.
At this point, the user can manipulate the model in manual mode using the 2-D or 6 DOF mouse.

When the simulated annealing algorithm is running, the model is continuously drawn in the latest estimated position, the effect of which is that the model is drawn as an animated figure which initially jitters around and then jockeys into final position. This animation allows the user to immediately see whether the fitting algorithm is converging to the correct solution, and if not, to take action to correct the problem. In the meantime, the simulated annealing algorithm automatically reduces the temperature to a fixed percentage of the previous value. The temperature is defined to be a number between zero and 100 . After the temperature has fallen to a sufficiently low level, the model stabilizes around its final position.

There are two techniques with which the user can interact with the system to provide guidance and constraints. These techniques, called traded control and shared control, draw again upon the paradigm of supervisory control [1]. In traded control, the user and the system each take turns controlling the pose of the model. We have found this to be particularly useful when the model fitting algorithm gets stuck in a local minimum. The user can immediately see that the model (shown as a graphical overlay on the image of the scene) has settled into an incorrect pose. By moving the mouse, the operator takes control of the model from the system, and can move it towards the correct pose. Releasing the mouse allows the system resume the model fitting algorithm. A small movement toward the correct pose is often enough to push the model out of the local minimum and allow it to automatically find the correct pose.

In shared control, the user controls some of the degrees of freedom, while simultaneously the system controls the other degrees of freedom. The operator can choose any of three different modes of shared control, as shown in Table 1 .

Table 1 Techniques in shared control.

\begin{tabular}{|c|c|}
\hline Mode & Constraints \\
\hline $\begin{array}{l}\text { Operator designates a } \\
2-D \text { point in the image }\end{array}$ & $\begin{array}{l}\text { Object center must lie along ray } \\
\text { emanating from the current viewpoint out } \\
\text { in the direction of the selected image point }\end{array}$ \\
\hline $\begin{array}{l}\text { Operator designates a } \\
\text { 3-D point }\end{array}$ & $\begin{array}{l}\text { Object center must be located at the } \\
\text { designated point }\end{array}$ \\
\hline $\begin{array}{l}\text { Operator controls a set } \\
\text { of orthogonal axes }\end{array}$ & $\begin{array}{l}\text { Object orientation must match the } \\
\text { specified axes }\end{array}$ \\
\hline
\end{tabular}

\section{Implementation}

This section describes the implementation of the model fitting system and informal evaluations performed with volunteer operators. The system was developed 
using the Silicon Graphics software package called "Open Inventor". which provides high level $\mathrm{C}++$ class libraries to create. display, and manipulate 3-D models.

The application displays the 3-D data points as small red boxes overlaid on the background image (the "left" image of the stereo set). The user selects a geometric primitive to fit to the data points. which is shown as a translucent model (Figure 3).

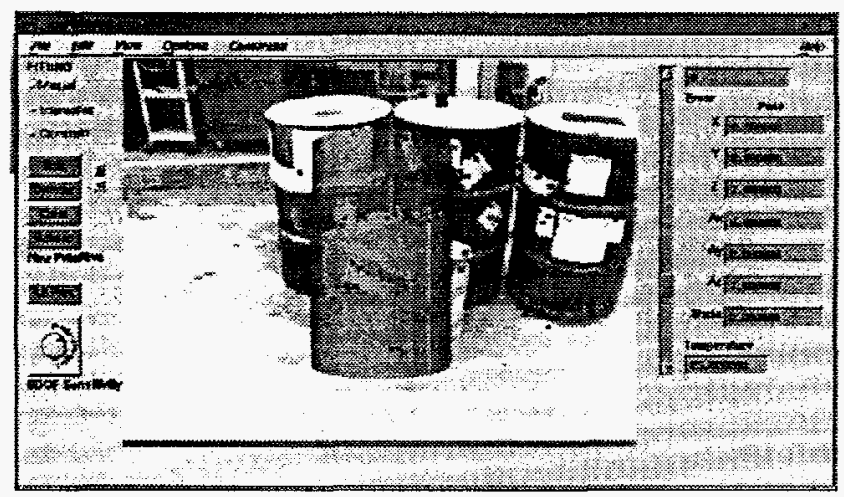

Figure 3 User interface for model fitting application. showing cylinder model in inirial pose

The uscr can manipulate the model in manual mode using the 2-D or 6 DOF mouse, or start the automatic model fitting (simulated annealing) algorithm. In either mode. the pose of the model is continuously displayed in the text fields on the right side of the window. Also displayed is the current "error" scorc, which represents the total distance of the 3-D points to the model The color of the model changes from shades of red to green as the error decreases.

When the simulated annealing algorithm is running, it gradually reduces the temperature at each iteration. The temperature is graphicaliy shown as a vertical bar immediatcly to the right of the image. The user can set the temperature manually by simply moving the bar up or down. We have found this to be useful when the model has appeared to reach the correct pose. In this case, we reduce the temperature to zero immediately to avoid any chance of the model escaping the correct solution. Figure 4 shows the model in its final pose.

An important part of this project was to quantify the benefits of the interactive object modeling techniques in terms of task completion time and model accuracy. We performed an informal evaluation, using four subjects that were not involved with the development of the system. Each subject was trained in the use of the 6 DOF mouse prior to evaluation, and allowed to practice fitting models on several sample scenes. Subjects were instructed that the first priority was to achieve the lowest error score, and the second priority was to minimize the time taken.

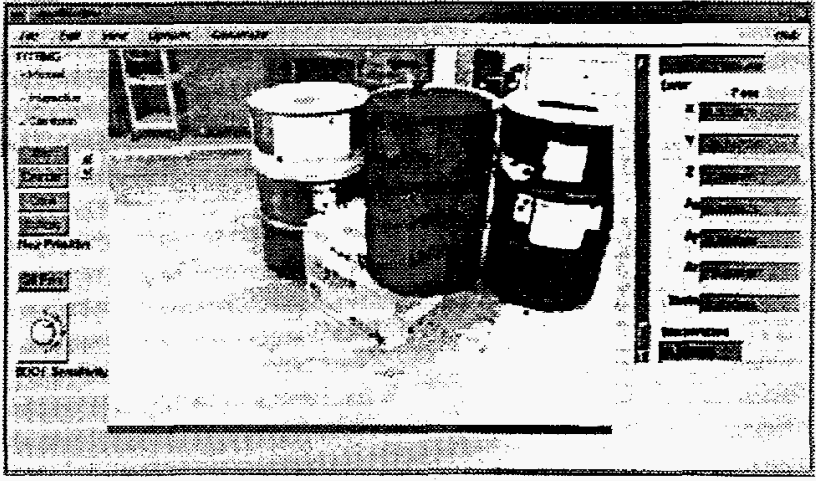

Figure 4 Final pose for a sample fitting run.

The subjects were then presented whth as set of 16 new scenes $(8$ synthetic scenes and 8 real scenes). The subjects fit models to 8 of these scenes manually and $x$ of the scenes interactively. The subjects then repeated the scenes, fitting models to the first 8 scencs interactively and the second 8 scenes manually. The crror sore ark pose results from these tasks were then recurded when the subject deemed the fit was acceptable

The cumulative arerage resuls are whun in rable? In this table. pose error was meinured mom only the synthetic scenes (since we did nom have erond truth pose data for the real sceness. Pose uncntation error was determined by measuring the angular devation of an axis of the model from the known ground truth axis direction From the results, it is evident that interactive htung was far superior 10 manual fitting in task ume and shghtly better in accuracy

Table 2 Summary of evaluation results.

\begin{tabular}{|c|c|c|c|}
\hline & Manual & Interactive & improvenen \\
\hline Task Tims & $83 \mathrm{sec}$ & $30 \mathrm{sec}$ & $53 \sec \left(00^{\circ} \mathrm{c}\right.$ \\
\hline Pose Transiation Error & $1.1 \mathrm{~cm}$ & $0.9 \mathrm{~cm}$ & $02 \mathrm{~cm}(1 \mathrm{~g})$ \\
\hline Pose Orientation Error & 2.3 & 10 & is $\left[57^{\circ}\right.$, \\
\hline Fit Error Score & 527 & 485 & $\left.4288^{\circ}\right)$ \\
\hline
\end{tabular}

We also compared the interactive fitung mode to purely automatic mode. Four fully automated (i.e.. no user interaction) runs from the default starling position were done for each of the 8 synthetic seenes. The system produced a correct result (almost identical on interactive runs for the same scenes) on only 16 out of 32 of these runs, or only $50 \%$ of the time. In other words, hall the time it was unable to escape local minma.

\section{Conclusions}

We have developed an interactive systom for fitting models to range data and have demonsirated its effectiveness (in terms of task time and accuracy) in 
preliminary evaluations with human operators. Unlike purely manual or purely autonomous systems, our interactive system combines the best attributes of each to create a system that has high operator productivity as well as high flexibility and robustness. Similar to supervisory control, we integrate human intelligence and machine intelligence to create a more powerful system that uses the best elements from each component. The human provides high level reasoning and overall guidance; the computer provides quantitative analysis and repeatable operation.

Our system can use extremely sparse range data (about $50-100$ points per object), can tolerate occlusions, and work in cluttered scenes. Through a combination of traded and shared control, the operator supervises the creation of models of objects in the scene. The system uses an optimization technique called simulated annealing for fitting primitive 3-D object models to the range data.

\section{Acknowledgments}

The authors wish to acknowledge the contributions of Torsten Lyon, Khoi Nguyen, Doug Swartzendruber, and Rex Rideout, and the volunteer test subjects. We also wish to thank to Mark McKay of INEL for his guidance and assistance in this project. Finally, we wish to thank Charles Little of Sandia National Labs for providing us with structured light range data.

\section{References}

[1] T. B. Sheridan, Telerobotics, automation, and human supervisory control, Cambridge, Massachusetts, MIT Press, 1992.

[2] S. Lee, "Intelligent Sensing and Control for Advanced Teleoperation," IEEE Control System Magazine. Vol. 13, No. 3, pp. 19-28, 1993.

[3] M. J. McDonald and R. D. Palmquist, "Graphical programming: On-line robot simulation for telerobotic control," Proc. of International Robots and Vision Automation Conference, pp. 22-59,22-73, 1993.

[4] J. Wagner, "Interactive computer-enhanced remote viewing system," in Innovation Investment Area Technology Summary, vol. DOE/EM-0146P, 1994, pp. 85-86.

[5] D. Cannon, G. Thomas, C. Wang, and T. Kesavadas, "A virtual reality based point-and-direct robotic system with instrumented glove," Intll J. of Industrial Engineers Applications and Practice, Vol. 1, No. 2, pp. 139-148,

[6] A. Leonardis, A. Gupta, and R. Bajcsy, "Segmentation of Range Images as the Search for Geometric Parametric Models," International Journal of Computer Vision, Vol, 14, No. 3, pp. 253-277, 1995.
[7] Y. Roth-Tabak and R. Jain, "Building an Environment Model Using Depth Information," Computer Magazine (IEEE). Vol. 22, No. 6, pp. 85-90, 1989.

[8] R. Bajcsy and F. Solina, "Three dimensional object representation revisited," Proc. of First Int'l Conf on Computer Vision, IEEE Computer Sociely, London, pp. 231-240, 1987.

[9] T. O'Donnell, T. Boult, X. Fang, and A. Gupta, "The extruded generalized cylinder: A deformable model for object recovery," Proc. of IEEE Conf. Computer Vision and Pattern Recognition, pp. 174-181, 1994.

$[10] \mathrm{K}$. Wu and M. Levine, "Recovering parametric geons from multiview range data," Proc. of IEEE Conf. Computer Vision and Pattern Recognition, pp. 159 166, 1994.

[11] M. Hebert, R. Hoffman, A. Johnson, and J. Osborn, "Sensor-Based Interior Modeling," Proc. of Robotics and Remote Systems, American Nuclear Society, Monterey, CA, pp. 731-737, 1995.

[12] W. E. L. Grimson, et al, "An automatic registration method for frameless stereotaxy, image guided surgery, and enhanced reality visualization," Proc of Computer Vision and Pantem Recognition, IEEE, pp. 430-436, 1994.

[13] P. J. Besl and N. D. McKay, "A Method for Registration of 3-D Shapes." IEEE Transactions on Pattem Analysis and Machine Intelligence. Vol. 14, No. 2, pp. 239-256. 1992.

[14] E. D. Dickmanns and V. Graefe, "Applications of Dynamic Monocular Machine Vision," Machine Vision and Applications, Vol. 1, No. pp. 241-261, 1988.

[15] S. J. Gee and A. M. Newman, "RADIUS: Automating Image Analysis Through Model-Supported Exploitation," Proc. of DARPA Image Understanding Workshop, Morgan Kaufmann, pp. 185-196, 1993.

[16] S. Heuel and R. Nevatia, "Including Interaction in an Automated Modeling System," Proc. of Image Understanding Workshop, ARPA, Palm Springs, pp. 429-434, 1996.

[17] P. V. Fua and Y. G. Leclerc. "Model driven edge detection," Proc. of DARPA Image Undersianding Workshop, Morgan Kauinann, pp. 1016-1021. 1988.

[18] N. Ayache and F. Lustman, "Fast and reliable passive trinocular stereo vision." Proc. of First Intl. Conf. Computer Vision, IEEE, pp. 422-427, 1987

[19] W. H. Press, S. A. Teukolsky, W. T. Vetterling, and B. P. Flannery, Numerical Recipes in $C$, 2nd ed., Cambridge University Press, 1992.

[20] S. Kirkpatrick, J. C.D. Gelatt, and M. P. Vecchi, "Optimization by Simulated Annealing." Science, Vol. 220, No. 4598 , pp. $671-680,1983$.

[21] K. H. Hoffman and P. Salamon, "The Optimal Simulated Annealing Schedule for a Simple Model," Joumal of Physics A: Mathematical and General, Vol, 23, No. 15 , pp. 3511-3523, 1990. 